\title{
Monte Carlo-based Tolerance Study of an End-fed Resonant Slotted Waveguide Linear Array Antenna
}

Lácides Ripoll-Solano

\author{
Luis Torres-Herrera
}

\author{
Manuel Sierra-Pérez
}

\begin{abstract}
This article presents a Monte Carlo-based analysis of the effects that random manufacturing errors produce on the radiation diagram of resonant slotted waveguide linear antennas. Positional and dimensional errors on the slots are considered. The study is performed using a tool implemented in MATLAB based on the Taylor synthesis of antenna parameters and the analysis of arrays and aperture models. The tool assesses the impact of the different errors on the radiation pattern. The results show that slot dimension errors have a dominant effect on the pattern over the variation on the slot position in the waveguide. A prototype of the studied antenna was build and its radiation pattern measured against the predicted mean and extreme tolerance margins. The results obtained are satisfactory, thus validating the implemented tool.
\end{abstract}

Keywords-antenna, slot, resonant, waveguide, tolerance, Monte Carlo

\section{INTRODUCCIÓN}

La síntesis de diagramas de radiación consiste en calcular el mejor conjunto de excitaciones en amplitud de señal $a_{n}$ tales que para un conjunto dado de posiciones $\left(x_{n}, y_{n}, z_{n}\right)$ de elementos del array, el diagrama de radiación o "factor de array" $F(\theta, \phi)$, se aproxime lo mejor posible a una forma deseada, de manera que se verifiquen determinadas especificaciones impuestas en la etapa de diseño [1]. Esto es posible sobre la premisa de que las excitaciones individuales puedan ser implementadas y operadas con absoluta precisión. Sin embargo en las antenas de ranuras en guías de ondas las excitaciones reales son afectadas por errores de fabricación mecánicos, lo cual resulta en una distorsión del diagrama de radiación. Por lo tanto, los diseñadores necesitan determinar la influencia que estos errores tienen sobre el diagrama de radiación y particularmente sobre el Nivel del Lóbulo Primario a Secundario (NLPS) para realizar correctivos en los procesos de fabricación.

El impacto de errores aleatorios en la geometría y posicionamiento de arrays de antenas han sido estudiados desde los años cincuenta. Por ejemplo, [2]-[4] consideraron los errores en cada elemento como variables aleatorias de distribución normal. Ruze [2] estudió el efecto de los errores aleatorios en las corrientes de excitación sobre el diagrama de radiación. Hsiao [3] encontró una relación entre el máximo NLPS y los errores aleatorios utilizando un método estadístico. Bailin y Ehrlich [4] determinaron los efectos físicos que resultan de los errores aleatorios en un array de ranuras lineal y determinaron teóricamente su impacto sobre el diagrama de radiación. Los errores son modelados con distribución normal y aplican a la longitud y posición de las ranuras, pero no se consideraron los efectos individuales de cada tipo de error sobre el NLPS. Por otro lado, mas recientemente en 2005, Lee [5] utilizó el método de Monte Carlo para encontrar la tolerancia de los errores en cada elemento del array de manera que con alta probabilidad, la fabricación de la antenna pudiera cumplir con las especificaciones del diagrama de radiación objetivo. Similarmente, Rengarajan, Zawadzki y Hodges [9] utilizaron técnicas de Monte Carlo para estudiar los efectos de la tolerancia y de modelamiento en antenas de radiometría con lóbulos laterales bajos sobre diferentes regiones angulares, donde obtuvieron un $4 \%$ de ancho de banda.

\section{Metodología}

Los diagramas de radiación de los arrays son determinados por los tipos de elementos individuales utilizados, sus orientaciones, posiciones, y las amplitudes y fases de las corrientes de excitación. Si $a_{n}$ representa los coeficientes de excitación ideales, tanto en amplitud como en fase, entonces el diagrama de radiación deseado $F_{\text {deseado }}$ para un array lineal puede ser expresado por:

$$
F_{\text {deseado }}(\theta)=\sum_{n=1}^{N} a_{n} e^{j \cdot n \cdot k \cdot d \cdot \cos \theta}
$$

donde $a_{n}$ son los coeficientes de excitación ideales, $d$ es la separación entre los elementos, $k$ es el número de onda del espacio libre y $\theta$ es el ángulo con respecto al eje del array. Si existe un error en amplitud y fase en los coeficientes de excitación, denotado como $\epsilon e^{j \cdot \alpha}$, el diagrama de radiación se distorsiona. Para errores aleatorios linealmente independientes, tanto en amplitud como en fase, el diagrama de radiación puede ser expresado como:

$$
F_{\text {error }}(\theta)=\sum_{n=1}^{N} a_{n} \epsilon_{n} e^{j \cdot \alpha_{n}} e^{j \cdot n \cdot k \cdot d \cdot \cos \theta}
$$

donde $\epsilon_{n}$ y $\alpha_{n}$ representan los errores en amplitud y fase, respectivamente.

En este trabajo se usó una forma común de array de guía de onda de ranuras la cual consiste de $i$ ranuras longitudinales cortadas en la pared ancha de una guía de onda rectangular. 


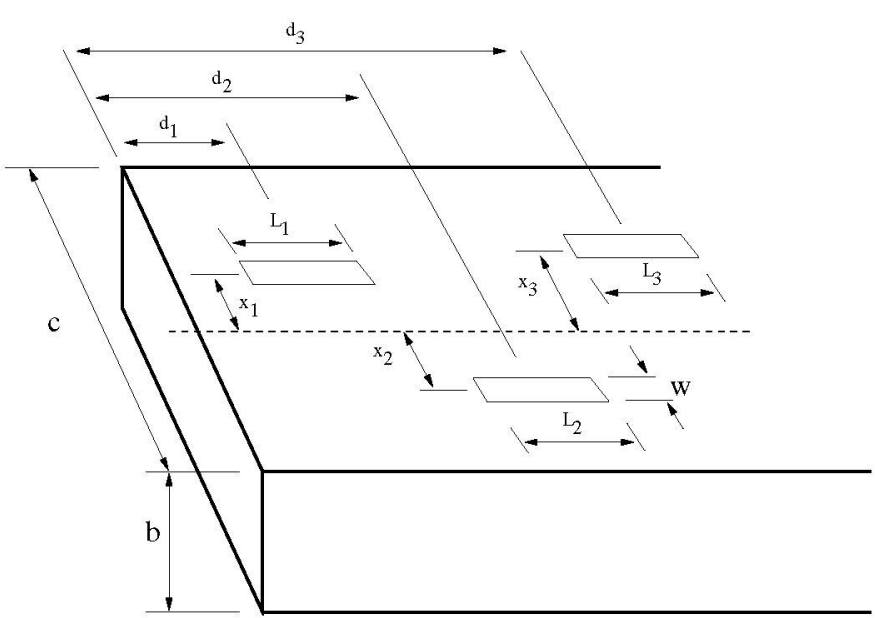

Fig. 1: Esquema del array de ranuras.

$\mathrm{Al}$ excitar esta guía con el modo dominante $T E_{10}$, la corriente fluye sobre las paredes de la guía. Los flujos de corriente interceptan las ranuras, que se encuentran cada una a una distancia particular $x_{i}$ del eje de la guía, causando la excitación de las ranuras y con ello la producción de radiación directiva. Los arrays resonantes son diseñados para operación broadside, y para obtener dicha radiación, todas las ranuras deben ser excitadas en fase, lo cual se consigue espaciando las ranuras $\lambda_{g} / 2$ y alternando su ubicación alrededor del eje central de la guía, como se observa en la Fig. 1. Sin embargo, cuando la guía es perforada para hacer las ranuras, se introducen errores aleatorios que distorsionan parámetros físicos como el espaciamiento entre ranuras, su longitud y la separación con respecto al eje. Esto repercute en la variación de la amplitud y la fase de radiación de cada ranura. Una aproximación razonable es que dichas variaciones generan errores que son independientes entre sí y distribuidos de manera normal alrededor de su media, que corresponden a los valores de diseño. Hay que tener en cuenta que a pesar de que el array puede presentar simetrías, dos ranuras que se encuentren ubicadas a igual distancia con respecto al centro no necesariamente presentarán los mismos errores.

\section{A. Antena seleccionada}

El estudio de tolerancia presentado en este trabajo se realiza sobre el diseño de la antena de ranuras lineal propuesto por Ripoll et al. in [6]. El diseño se realizó con los parámetros mostrados en la Tabla I. La distancia $x_{i}$ de separación de cada ranura con respecto al eje de la guía se encuentra en la Tabla II.

La Fig. 2 muestra la antena construida, abierta a la izquierda y cerrada a la derecha, con las ranuras sólo en la cara superior y exhibiendo simetría entre la $1^{\text {era }}$ y la $11^{a v a}$, la $2^{d a}$ y la $10^{\text {ma }}$, etc. Dejando la $6^{t a}$ como ranura central del array.

La Fig. 3 muestra la comparación entre los diagramas de radiación: 1) simulado con MATLAB ${ }^{\circledR}$,2) simulado con el CST Microwave Studio ${ }^{(}$, y 3) el obtenido experimentalmente a través de mediciones. Se aprecia una muy buena correlación entre los valores teóricos y el experimental. Esto tiene dos
TABLA I: PARÁMETROS DE DISEÑO DE LA ANTENA

\begin{tabular}{|c|c|}
\hline Parámetro & Valor \\
\hline \hline Frecuencia & $12 \mathrm{GHz}$ \\
\hline NLPS & $20 \mathrm{~dB}$ \\
\hline Número de ranuras & 11 \\
\hline Ancho de ranura $w$ & $1 \mathrm{~mm}$ \\
\hline Longitud de ranura & $12.5 \mathrm{~mm}$ \\
\hline Distribución de corrientes & Taylor de un parámetro \\
\hline Guía de onda & WR-75 \\
\hline Ancho interior & $19.05 \mathrm{~mm}$ \\
\hline Alto interior & $9.5 \mathrm{~mm}$ \\
\hline
\end{tabular}

TABLA II: Posición DE LAS RANURAS CON RESPECTO AL EJE CENTRAL DE LA GUÍA

\begin{tabular}{|c|c|c|c|c|}
\hline Ranura & $x_{i}(\mathbf{m m})$ & Ranura & $x_{i}(\mathbf{m m})$ \\
\hline \hline 1 & 0.9510 & 7 & 2.7180 \\
\hline 2 & 1.4790 & 8 & 2.4250 \\
\hline 3 & 1.9920 \\
\hline 4 & 2.4250 & 9 & 1.9920 \\
\hline 5 & 2.7180 & 10 & 1.4790 \\
\hline 6 & 2.8210 & - & 0.9510 \\
\hline \multicolumn{2}{|c|}{} \\
\hline
\end{tabular}

consecuencias favorables. Por un lado, los resultados del modelo de MATLAB, que es más sencillo y apegado a la teoría, son aproximados dentro de un margen de alrededor $3 \mathrm{~dB}$, con el producido con el CST, que toma en cuenta un mayor número de parámetros de construcción. Esto valida el uso del modelo sencillo de MATLAB. Por otro lado, el resultado experimental apoya ambas simulaciones. Aunque en principio las medidas muestran un lóbulo secundario ligeramente más bajo (más favorable) que las simulaciones, en general para los lóbulos laterales más significativos, se nota una concordancia aceptable. En los extremos de la gráfica las mediciones resultan un tanto distintas, pero los niveles de radiación en estos ángulos son despreciablemente bajos.

Numerosas simulaciones de Monte Carlo permiten apreciar que errores de fabricación en los siguientes parámetros de diseño no impactan significativamente sobre el diagrama de radiación y pueden ser despreciados:

a) Variaciones en la impedancia del array, dado su insensibilidad a diferentes errores longitudinales y posicionales, como lo presenta Bailin [4].



Fig. 2: Antena de ranuras construida. 




Fig. 3: Comparación de los diagramas de radiación obtenidos con el modelo teórico en MATLAB, en el simulador CST y la medición experimental.

b) E1 cambio en la fase producido por la aleatoriedad en el desplazamiento transversal de las ranuras $x_{i}$, por ser un error de segundo orden comparado al cambio en la fase debido a variaciones en la longitud de las ranuras $l_{i}$, también indicado por Bailin [4].

c) El ancho de las ranuras $w$, pues no se encontraron diferencias apreciables en la radiación para valores desde $\lambda / 400$ hasta $\lambda / 40$.

\section{B. Selección de errores relevantes}

Con base en criterios avalados por la literatura y resultados parciales al aplicar el método de Monte Carlo, se reconocen los siguientes tipos de error de fabricación como los que tienen un impacto relevante en la forma del diagrama de radiación. Por lo tanto las simulaciones solo tendrán en cuenta:

a) Variación en la amplitud debido a la aleatoriedad en la posición transversal de las ranuras $x_{i}$.

b) Variación en la fase debido a la aleatoriedad en la posición longitudinal de las ranuras $d_{i}$.

c) Variación en la fase debido a la aleatoriedad en la longitud de las ranuras $l_{i}$.

Para el primer tipo de error, Stern [7] demostró que las ranuras pueden ser modeladas como admitancias complejas en paralelo donde la conductancia normalizada (parte real de la admitancia) de una ranura longitudinal cortada en una guía de onda operando en el modo $T E_{10}$ viene dada por:

$$
g=2.09 \frac{\lambda_{g}}{\lambda} \frac{c}{b} \cos ^{2} \frac{\pi \lambda}{2 \lambda_{g}} \sin ^{2} \frac{\pi x}{c}
$$

donde $c$ y $b$ son el ancho y el alto de la guía de onda rectangular, $\lambda$ es la longitud de onda en el espacio libre, $\lambda_{g}$ es la longitud de onda en la guía y $x$ es el desplazamiento de la ranura desde el eje de la guía. El coeficiente de excitación relativo de una ranura $a_{n}$ es proporcional a $\sqrt{g}$.
Para el segundo tipo de error, la fase relativa entre las señales de dos ranuras consecutivas, vienen determinadas por dos términos: 1) la diferencia entre las longitudes del trayecto en el espacio libre al punto donde se desea calcular el campo, y 2) la diferencia en el trayecto entre los radiadores a lo largo del array. La última diferencia es reducida alternando las ranuras sobre lados opuestos del eje de la guía. Quedando la diferencia de fase como:

$$
\phi_{g}=\frac{2 \pi d}{\lambda} \sin \theta+\frac{2 \pi d}{\lambda_{g}}-\pi
$$

donde $\lambda$ es la longitud de onda del espacio libre, $\lambda_{g}$ es la longitud de onda en la guía, $d$ es la separación entre ranuras, y $\theta$ es el ángulo con respecto al eje del array, de acuerdo con Bailin [4].

Para el tercer tipo de error, la magnitud de la variación en la fase se puede ver como una función de un error en la longitud de la ranura, determinada experimentalmente por Lee [5], y dada por:

$$
\phi_{L}=\frac{K\left(L_{0}-L\right)}{L_{0}}
$$

donde $K=5 \pi$ es la pendiente de valores determinados experimentalmente por Stegen [8], $L_{0}$ es la longitud de resonancia de una ranura y $L$ es la longitud real de la ranura. La fase total de la radiación puede ser obtenida como:

$$
\phi_{\text {Total }}=\phi_{d}+\phi_{L}
$$

\section{Simulaciones de Monte Carlo}

Esta sección se divide en tres partes. La primera presenta los modelos asumidos para la aleatoriedad en el posicionamiento y dimensiones de los elementos del array ocurrida en la fabricación de la antena. La segunda muestra las simulaciones de Monte Carlo con una descripción estadística de las distribuciones del diagrama de radiación en términos de medias y percentiles extremos, y el grado de impacto sobre el NPLS, que es el indicador más importante en el diseño de la antena. La tercera realiza una exploración de la sensibilidad a cada una de los tres tipos de error estudiados, indicando cuál de ellos tiene mayor impacto en el deterioro del diagrama del radiación. Finalmente, se comparan estos resultados de márgenes para mejores y peores casos del patrón de radiación, con los valores medidos experimentalmente en la antena construida.

\section{A. Modelos de tolerancias}

En la Sec. II-B se presentaron los tres tipos de errores cuyo impacto se va a estudiar con el método de Monte Carlo. A continuación se presentan los modelos empleados, destacando las variables aleatorias para cada una de las $i$ ranuras, y además el significado de la tolerancia (variabilidad) de fabricación.

1) Posición transversal de ranuras: el primer error es la posición transversal del centro de la ranura con respecto al eje longitudinal que está sobre la cara ranurada de la guía. Las posiciones de diseño original se denotan con $x_{i}^{\text {diseño }} y$ están dadas en la Tabla II. Sobre estas posiciones se añade una variable aleatoria de distribución normal estándar escalada al nivel de errores considerados, denotado con $\sigma_{\text {error }}$ y adquire 
TABLA III: PARÁMETROS USADOS EN LA SIMULACIÓN DE COMPORTAMIENTO DE LA ANTENA

\begin{tabular}{|c|c|}
\hline Parámetro & Valor \\
\hline \hline Frecuencia & $12 \mathrm{GHz}$ \\
\hline NLPS & $20 \mathrm{~dB}$ \\
\hline Número de ranuras & 11 \\
\hline Ancho de ranura $w$ & $1 \mathrm{~mm}$ \\
\hline Longitud de ranura & $\lambda / 2=12.5 \mathrm{~mm}$ \\
\hline Distribución de corrientes & Taylor de un parámetro \\
\hline Tolerancia en los tres errores & $\pm 1 / 10 \mathrm{~mm}$ \\
\hline
\end{tabular}

valores de $1 / 10 \mathrm{~mm}$ o mayor. Este primer caso de tolerancia se denota $T_{1}$ y se define como:

$$
T_{1}: X_{i}=\mathrm{x}_{i}^{\text {diseño }}+\sigma_{\text {error }} \cdot \mathcal{N}(0,1)
$$

2) Posición longitudinal de ranuras: el segundo error es la posición longitudinal del centro de la ranura con respecto al eje longitudinal que está sobre la cara ranurada de la guía. Las posiciones de diseño original $\mathrm{d}_{i}^{\text {diseño }}$ vienen dadas por múltiplos impares de $\lambda_{g} / 4$, es decir, $\lambda_{g} / 4,3 \lambda_{g} / 4,5 \lambda_{g} / 4$, etc. Sobre estas posiciones se añade una variable aleatoria de distribución normal estándar escalada al nivel de los errores considerados, similar al primer caso, $\sigma_{\text {error }}$. Este segundo caso de tolerancia se denota $T_{2}$, y se define como:

$$
T_{2}: D_{i}=\mathrm{d}_{i}^{\text {diseño }}+\sigma_{\text {error }} \cdot \mathcal{N}(0,1)
$$

where

$$
\mathrm{d}_{i}^{\text {diseño }}=\frac{\lambda_{g}}{4}[1+2(i-1)]
$$

3) Longitud de las ranuras: el tercer error es la longitud de las ranuras hechas en la guía. La longitud original de diseño es simplemente $\lambda / 2$, y sobre este valor se añade una aleatoriedad

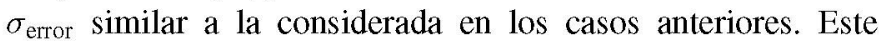
tercer caso de tolerancia se denota $T_{3}$, y se define como:

$$
T_{3}: L_{i}=\frac{\lambda}{2}+\sigma_{\text {error }} \cdot \mathcal{N}(0,1)
$$

B. Simulaciones con la herramienta implementada en MAT$L A B$

Las simulaciones fueron realizadas bajo las condiciones mostradas en la Tabla III. La Fig. 4 muestra a manera de ejemplo 30 diagramas de radiación obtenidos de simulaciones de Monte Carlo para el plano de $\phi=90^{\circ}$, donde se observa un caso crítico en el deterioro del NLPS deseado y las variaciones en los distintos lóbulos laterales tanto en sus ángulos de caída como en sus valores máximos.

Para el análisis de resultados completo, el estudio de Monte Carlo se realiza con 10.000 simulaciones de diagramas de radiación, obteniéndose la Fig. 5, donde se muestra el promedio de estas comparadas con el valor teórico.

La Fig. 6 muestra que la distribución de los NLPS obtenidos es aproximadamente normal. Se observa que para un valor de diseño del NLPS de $20 \mathrm{~dB}$, la media está alrededor de este valor y el $95 \%$ de los resultados son mayores a $17 \mathrm{~dB}$. Por otro lado, la Fig. 7 muestra las distribución de los niveles de

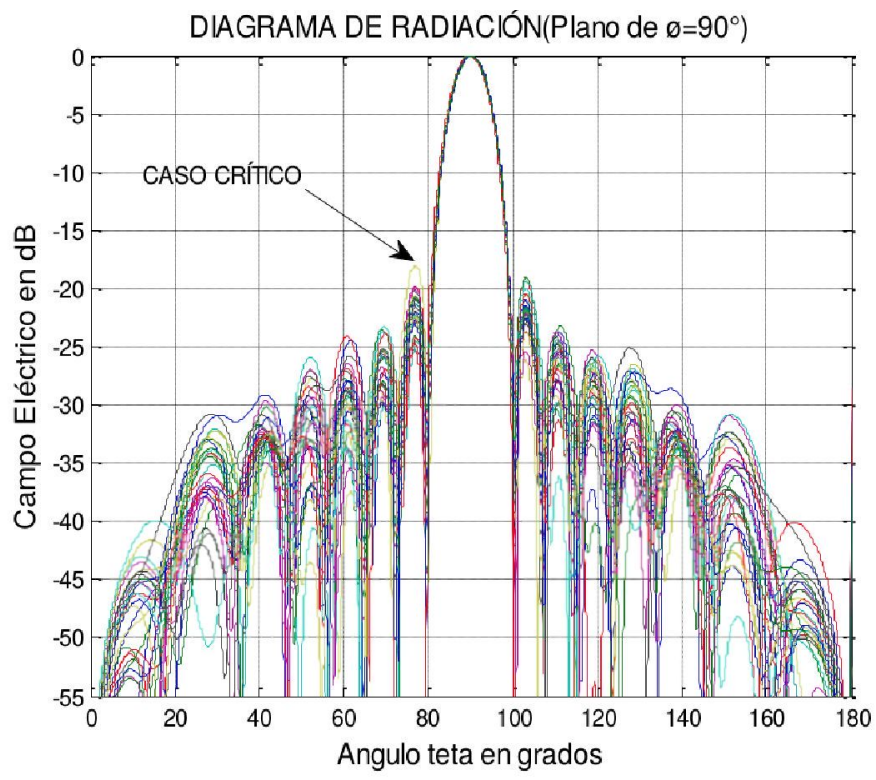

Fig. 4: Diagrama de radiación para 30 simulaciones $\left(\theta=90^{\circ}\right)$.

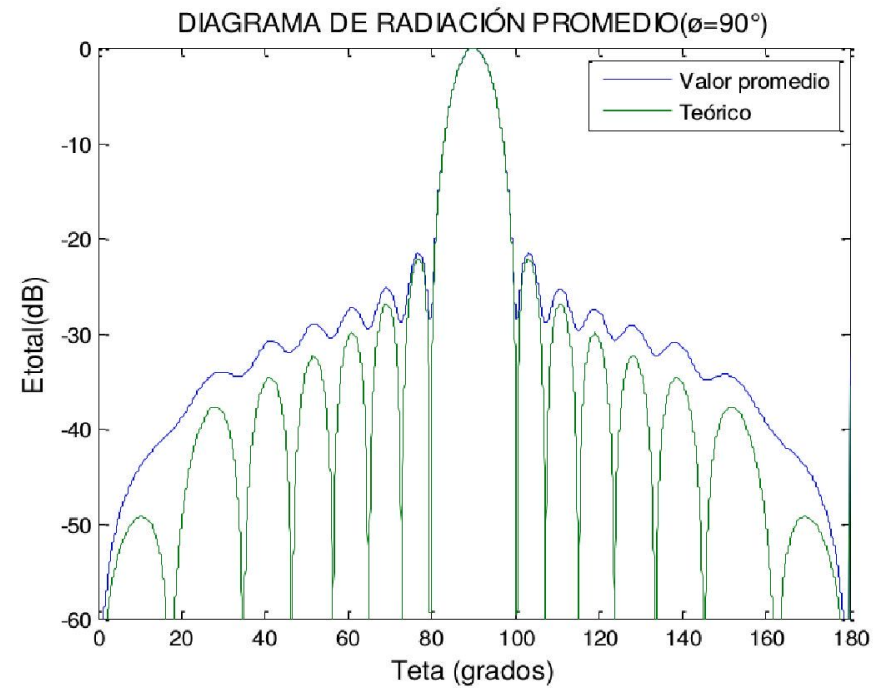

Fig. 5: Diagrama de radiación promedio y teórico $\left(\phi=90^{\circ}\right)$.

señal para $\theta=72^{\circ}$. La forma de la distribución es asimétrica con sesgo hacia valores menores de potencia. Debido a que en general para todo ángulo $\theta$, el $E_{\text {Total }}$ radiado sigue una distribución similar a la de la Fig. 7, se recurre a describir los márgenes por arriba y por debajo de la media del diagrama de radiación por medio de percentiles, en vez de número de desviaciones estándar.

Las primeras tres curvas de la Fig. 8 muestran los límites dados por el percentil 95, la media y el percentil 5 del total de los diagramas de radiación de la simulación Monte Carlo. El percentil 95 significa que el 96\% de los diagramas no superan esta frontera. Esto permite concluir que una fresadora con una tolerancia de $\pm 0.1 \mathrm{~mm}$ presenta un peor caso esperado dado por la gráfica del percentil 95. Se aprecia que se viola el valor objetivo de diseño del NLPS por aproximadamente 3 


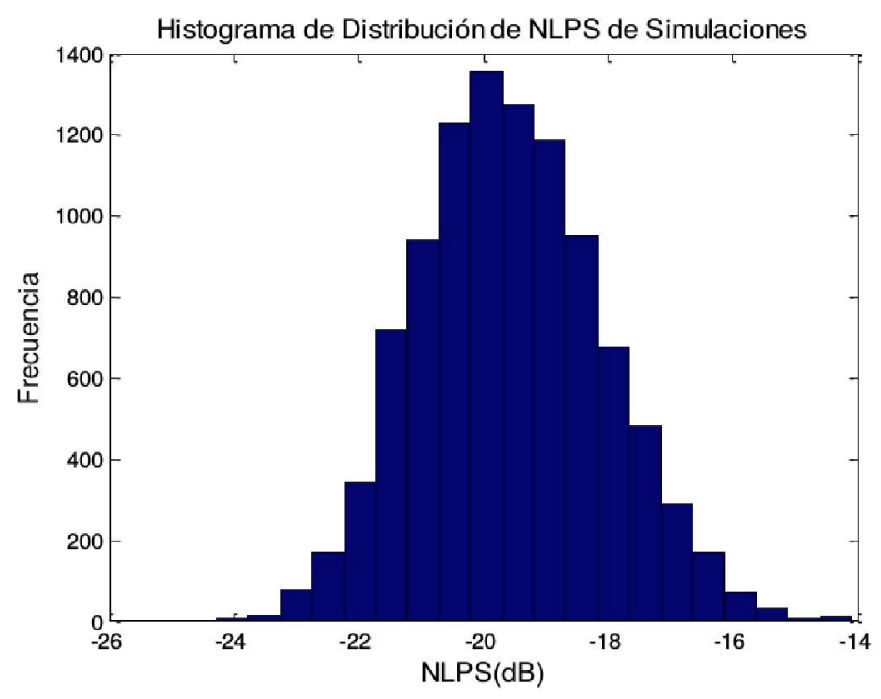

Fig. 6: Distribución del NLPS.

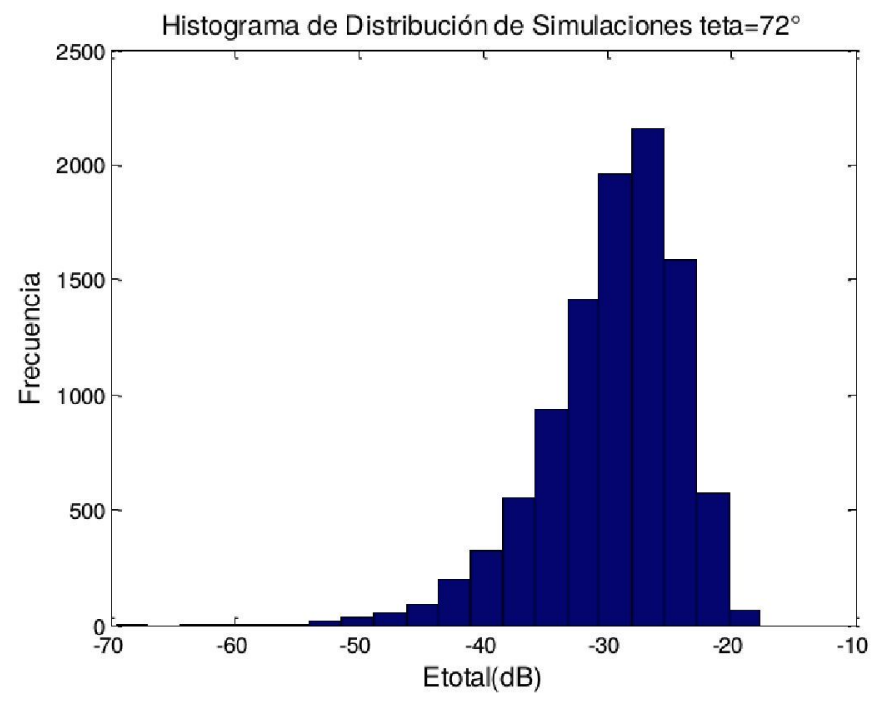

Fig. 7: Distribución de $E_{\text {Total }}$ para $\theta=72^{\circ}$.

dB, lo cual coincide con lo mostrado en la Fig. 6. La curva del percentil 5 representa un escenario optimista e indica el mejor diagrama posible alcanzable en las condiciones de tolerancia simuladas. La curva de la media es un valor relativamente cercano al teórico, como se mostró en Fig. 5.

\section{Análisis de Sensibilidad}

Para evaluar el impacto de cada tipo de error en el NLPS, se incremento 10 veces alternadamente cada uno de estos, y se observó el impacto en el NLPS. Para esto se realizó un cálculo para un array de 11 ranuras, NLPS de diseño de 20 $\mathrm{dB}$, frecuencia de operación de $12 \mathrm{GHz}$, y una tolerancia de $\pm 0.1 \mathrm{~mm}$ en todos los tres tipos de error presentados en la Sec. III-A.

Los resultados se presentan en la Tabla IV. Se observa que el error longitudinal de las ranuras $T_{3}$, tiene el efecto más dominante sobre los demás, decrementando el NPLS en hasta



Fig. 8: Diagrama de radiación experimental comparado con los límites de dispersión del dados por el valor medio y los percentiles $5^{\text {to }}$ y $95^{\text {avo }}\left(\phi=90^{\circ}\right)$.

TABLA IV: IMPACTO DEL TIPO DE ERROR EN LA DISTORSIÓN DEL NLPS $N P L S_{\text {diseño }}=20 \mathrm{~dB}, f=12 \mathrm{GHz}$

\begin{tabular}{|c|c|c|c|c|}
\hline \multirow{2}{*}{ Caso } & $T_{1}$ & $T_{2}$ & $T_{3}$ & $\bar{N}$ \\
\cline { 2 - 4 } & \multicolumn{2}{|c|}{ todas en $1 / 10 \mathrm{~mm}$} & $(\mathrm{~dB})$ \\
\hline \hline 1 & 1 & 1 & 1 & 19.4 \\
\hline 2 & 1 & 1 & 10 & 3.15 \\
\hline 3 & 1 & 10 & 1 & 16.5 \\
\hline 4 & 1 & 10 & 10 & 13.4 \\
\hline 5 & 10 & 1 & 1 & 12.2 \\
\hline 6 & 10 & 1 & 10 & 2.8 \\
\hline 7 & 10 & 10 & 1 & 3.1 \\
\hline 8 & 10 & 10 & 10 & 2.8 \\
\hline
\end{tabular}

$17 \mathrm{~dB}$; seguido de la posición transversal de los centros de las ranuras $T_{1}$, disminuyendo el NPLS hasta $8 \mathrm{~dB}$. El impacto del error $T_{2}$, el error en la posición longitudinal de las ranuras tan solo resta de 3 a $4 \mathrm{~dB}$ al NPLS

Adicionalmente se encontró que en los casos 2, 4, 6 y 8 de la Tabla IV, la dirección apuntada por el lóbulo principal presenta un corrimiento. En el caso 3, los lóbulos laterales comprendidos entre 90 y 180 grados presentan una mejora en su NLPS. En el caso 5 se presentó un ensanchamiento del lóbulo principal, y en el caso 7 hay tanto ensanchamiento como corrimiento de la direccion del lóbulo principal.

\section{Construcción del array y resultados}

El caso simulado en MATLAB en la Sec. III-B se construyó como se muestra en la Fig. 2. Sus características son: guía de onda WR-75 de cobre, 11 ranuras, la excitación de ranuras sigue una distribución de Taylor de un parámetro, frecuencia de operación $12 \mathrm{GHz}$, distancia entre ranuras adyacentes $\lambda_{g} / 2$ que corresponde a $16,598 \mathrm{~mm}$. La última ranura está a una distancia del borde cortocircuitado de $24,897 \mathrm{~mm}$. Para el corte de las ranuras se utilizó un sistema de fresado que presentó 
una tolerancia en la posición y la longitud de las ranuras de $\pm 0.1 \mathrm{~mm}$.

La cuarta curva de la Fig. 8 muestra que el diagrama de radiación experimental sigue muy bien al diagrama promedio y se encuentra entre los márgenes esperados, especialmente para los primeros lóbulos laterales. El percentil 95 puede ser tomado como el peor caso de error esperado. Esto demuestra que el estudio de tolerancia permite anticipar al fabricante las situaciones de peor caso. Si esta satisface los requerimientos de diseño, es altamente probable que al fabricar la antena, se tenga un resultado mejor que dicho caso. Es de esperar que resulte para los primeros lóbulos, alrededor del valor medio esperado.

\section{CONCLUSIONES}

Se ha mostrado como los errores aleatorios en la construcción de un array de ranuras longitudinales puede afectar el nivel de lóbulo primario a secundario (NLPS). Se encontró que los errores longitudinales presentan un efecto dominante sobre los demás. El estudio de tolerancia puede brindar garantías estadísticas de los resultados al indicar el peor caso (percentil 95). Si este caso cumple con los requerimientos de diseño, entoces se tiene certeza de que el proceso de fabricación será exitoso aún cuando las tolerancias de las máquinas ocasiones algunas distorsiones al diagrama de radiación. Se observa que para que el $95 \%$ de la producción cumpla con el requerimiento de NLPS, se debe exigir al diseño un valor objetivo de aproximadamente $3 \mathrm{~dB}$ más exigente, si los errores se mantienen alrededor de $\pm 0.1 \mathrm{~mm}$. Si se debe escoger preservar un error pequeño en detrimento de los demás, se debe tratar de minimizar el error $T_{3}$, el error en la longitud de las ranuras.

\section{REFERENCIAS}

[1] A. Cardama Aznar, L. Jofre Roca, J. Rius Casals, J. Romeu Robert, and S. Blanch Boris, Antenas, 1st ed. Barcelona: Alfaomega, 2000.

[2] J. Ruze, "The effect of aperture errors on the antenna radiation pattern," Nuovo Cimento (Suppl.), vol. 9, no. 3, pp. 364-380, 1952.

[3] J. K. Hsiao, "Normalized relationship among errors and sidelobe levels," Radio Sci., vol. 19, pp. 292-302, Jan. 1958.

[4] L. Bailin and M. Ehrlich, "Factors affecting the performance of linear arrays," Proc. IRE, vol. 41, pp. 235-241, Feb. 1953.

[5] J. Lee, Y. Lee, and H. Kim, "Decision of error tolerance in array element by the Monte Carlo method," IEEE Trans. on Antennas and Propagation, vol. AP-53, no. 4, pp. 1325-1331, Apr. 2005.

[6] L. Ripoll, M. Sierra, and D. Pardo, "Design, simulation and test of a slot antenna array using one parameter Taylor synthesis in the GHz range," IEEE Latin America Trans., vol. 13, no. 10, pp. 3210-3215, Oct. 2015.

[7] G. Stern and R. Elliott, "Resonant length of longitudinal slots and validity of circuit representation: Theory and experiment," IEEE Trans. on Antennas and Propagation, vol. 33, no. 11, pp. 1264-1271, Nov. 1985.

[8] R. Stegen, "Design of linear arrays," Proc. IRE - West Coast Meeting, Aug. 1951.

[9] S.Rengarajan, M. Zawadzkr, and R.Hodges, "Waveguide-slot array antenna designs for low-average-sidelobe specifications," IEEE Antennas and Propagation Magazine, vol. 52, no. 6, Dec. 2010. 